\title{
La migración de médicos latinoamericanos a Chile en las últimas décadas
}

JORGE LASTRA(1)

\section{INTRODUCCIÓN}

Varios miles de médicos latinoamericanos han migrado a Chile a partir de los noventa. Su presencia, que para muchos ha representado una solución a la falta de profesionales en la Atención Primaria, ha sido también un factor de conflicto, porque no pocos, especialmente de la orden médica, han criticado esta corriente migratoria. Tal como se ha afirmado en la prensa, este hecho ha sido comentado muy intensamente. En La Tercera del 24 de julio de 2001, se indicaba "regulación a ingreso de médicos extranjeros"; mientras ya con anterioridad, la Agrupación de Trabajadores de la Atención Primaria Municipalizada, pedía al Gobierno revisar los convenios internacionales de convalidación de carreras ${ }^{1}$.

Las estadísticas oficiales del Ministerio de Relaciones Exteriores, manifestaban que el año 2000, los médicos extranjeros que llegaron a nuestro país duplicaron el número de profesionales chilenos agresados ${ }^{1}$. Por su parte, Francisco León, de la Cepal, señalaba que: “... en el período 1990-1997 habrían sido autorizados a trabajar legalmente 1297 médicos, un sexto de los cuales, por reválida de títulos y el resto por convenios internacionales, habiéndose más que quintuplicado el número de autorizaciones durante ese período" 2 . Frente a esta misma situación, el Colegio Médico planteaba una posición de resguardo respecto del nivel técnico de estos profesionales, ya que -a su juicio- éste debía ser el parámetro exigible para cualquier médico chileno o extranjero. Y agregaba, que: "Tal pretensión es en resguardo de nuestra población y del prestigio de la profesión médica"3.

Sin embargo, para muchos otros actores, esta posición tenía que ver más bien con aspectos, donde la xenofobia y la preocupación por la competencia por el mercado del trabajo, aparecían como las verdaderas causas. Así, la prensa recogía las críticas que se le hacían a los médicos chilenos: "Alcaldes, Ministerio de Salud y trabajadores de la Salud municipalizada condenaron actitud de la orden gremial. Fuerte rechazo a xenofobia del Colegio Médico"l. Por último, los propios médicos extranjeros que trabajaban en Chile declaraban la falta de acogida de los profesionales para con ellos. Así, hicieron llegar a la prensa: "Una serie de críticas a la falta de solidaridad y sensibilidad social dada en una parte importante de los médicos locales"4. Este fenómeno social, cuantificable y frente al que gira una serie de actores, fue durante la segunda mitad de la década, un hecho muy documentado como problema y ha vuelto a estar presente con motivo de la actuación de un médico ecuatoriano, revelando diversas áreas de conflicto; fundamental a mi juicio es la contradicción de una norma creada en el marco de la integración de la década de los sesenta y el impulso dado a la migración por la globalización y las reformas a las políticas de salud chilena.

\footnotetext{
(1) Director del Servicio de Salud Metropolitano Central.jlastra@ssmc.cl
} 
Los fundamentos de esta hipótesis se encuentran en una revisión de varios antecedentes: el proceso de integración de los sesenta, cuando se firmó el Convenio Andrés Bello y que ha sido el acuerdo que ha posibilitado esta fuerte migración, los fenómenos de migración internacional, el impacto de la globalización en este mismo fenómeno y la inserción de nuestro país en el mundo.

\section{INTEGRACIÓN EN AMÉRICA LATINA EN LA ÉPOCA DE LA SUSTITUCIÓN DE IMPORTACIONES}

Uno de los principales acuerdos internacionales que ha generado impacto en la migración de médicos a nuestro país, ha sido el Convenio Andrés Bello. Este fue firmado el año 1972 y facilita el reconocimiento de los títulos profesionales universitarios, otorgados en alguno de los países firmantes en cualquiera de los otros participantes de este acuerdo. En su declaración, el Convenio Andrés Bello destaca su objetivo de: "Integrar educativa, científica, tecnológica y culturalmente a Bolivia, Colombia, Chile, Cuba, Ecuador, España, Panamá, Paraguay, Perú y Venezuela... creando un espacio cultural común". Y, postula en el fondo que: "La integración de América Latina y España a partir de la ciencia y la tecnología y la cultura es un sueño acariciado desde hace mucho tiempo"5.

Este convenio, forma parte de una serie de otros tratados que se produjeron en el contexto de un proceso de integración que se desarrolló enmarcado en las características del Continente Americano, durante el período de post guerra. Después de la Segunda Guerra Mundial, los Estados latinoamericanos buscaron caminos para su autodeterminación, a través de modelos propios para su desarrollo económico y político, y de una coordinación de las políticas económicas entre ellos.

La Comisión Económica de las Naciones Unidas para América Latina y el Caribe (Cepal), fundada en 1948, asumió a partir de los años cincuenta un papel protagonista en la integración regional, que se basaba en tres elementos interdependientes 6 .
1.Alterar su política económica exterior, con el objetivo de mejorar su situación desventajosa y periférica ante la economía mundial, a través de un movimiento integrativo subcontinental, como medio de disminuir las deterioradas relaciones de intercambio de sus materias primas con el capitalismo central.

2.El modelo de substitución de importación que debería impulsar el desarrollo económico a través del proceso de industrialización. Por esa razón, Cepal buscó a través de sus organizaciones, armonizar las políticas económicas, así como integrar los mercados latinoamericanos entre sí, como estrategia para superar la "dependencia económica secular" en relación a los países industrializados.

3.El componente diplomático debería fortalecer la capacidad de negociación frente a gremios internacionales. Cepal procuró, en los años sesenta, a través de la Comisión Especial de Coordinación, Cecla, articular posiciones homogéneas de la región, mediante gremios económicos multilaterales, como también en relación a los Estados Unidos, buscando articular en la región nuevos mecanismos para la modernización de los sectores de servicios capaces de conciliar el apoyo e interés del grupo de agentes políticos y económicos de la mayor amplitud posible. En este sentido, había un intensivo enlace de los países latinoamericanos a canales políticos de representación del "tercer mundo" en niveles internacionales.

A partir del marco que plantea la Cepal, se desarrollan en el continente diversas formas de integración económica, entre los años sesenta hasta los años ochenta ${ }^{7-9}$. Algunas de estas expresiones de Integración Interregional son: el Mercado Común de Centroamérica; en la región del Caribe el Caricom y en América del Sur: la Alac, Aladi, Grupo Andino, etc. La integración entre los países andinos es vista como la forma más intensa de integración regional entre países similares en relación a su desarrollo económico y tecnológico. El Acuerdo de Cartagena firmado en los años sesenta, abarcó 
no sólo sectores políticos, culturales y jurídicos, sino también creó un parlamento y un tribunal de justicia. A pesar de su carácter prometedor de la fase inicial, en los años ochenta, el Grupo Andino sufrió la misma crisis, de las demás formas integrativas de la región; resultado de la política exterior de las dictaduras que predominaron en el continente.

\section{LA MIGRACIÓN INTERNACIONAL}

Los movimientos de población siempre han estado presentes en la historia de la humanidad. Desde la existencia de la historia escrita, es posible constatar el desplazamiento de hombres, en respuesta al crecimiento demográfico, al cambio climático y a las necesidades económicas. Más adelante, las guerras y la formación de las fronteras y de las naciones y los Estados, han constituido nuevos motivos para las migraciones.

Todos los grandes episodios de la historia que han producido cambios de los espacios y del tiempo, como la circunnavegación de los océanos y la revolución industrial, se han transformado en condiciones promotoras de las corrientes migratorias. Lo mismo ocurre con la Segunda Guerra Mundial, después de la cual el volumen y el alcance de la migración aumentaron; afectando cada vez a más países y las naciones de inmigración recibían a personas procedentes de un amplio espectro económico, social y cultural. En todo caso, el período posterior a la Segunda Guerra Mundial, tiene dos ciclos. El primero hasta la crisis del petróleo en 1973, motivado por el auge económico se estimuló la migración a gran escala desde las regiones menos a las más desarrolladas. Junto con la "crisis del petróleo" y la depresión económica que la sigue, se produce el efecto contrario; de contención del flujo migratorio. El segundo ciclo de posguerra, comienza en la década de los ochenta y se mantiene activo desde entonces, con dimensiones sin precedentes. Dado estos antecedentes, que destacan el aumento a partir de los ochenta, coincidiendo con el fenómeno de la globalización. Castel señala que: "La migración internacional es parte integrante de la mundialización, que puede ser definida como una ampliación, profundización y aceleración de la interconexión mundial en todos los aspectos de la vida social contemporánea"10. Vale decir, la migración internacional es un proceso integrante de todos aquellos otros fenómenos que han constituido a la humanidad de nuestros días y que corresponde a la "interconexión mundial" que señala Castels.

Las causas de la migración son variadas y cubren diversas dimensiones de la vida social, como los aspectos económicos, políticos, culturales, demográficos, etc. En general, la primera causa considerada para la migración es la disparidad entre los niveles de ingreso, empleo y bienestar social. También son importantes otros patrones como los demográficos y el crecimiento de la fuerza laboral ${ }^{10}$. En todo caso la información de archivo no parece respaldar la creencia de que la pobreza es la gran causa de las migraciones. Más bien la migración laboral se origina en países con un nivel intermedio de desarrollo. Sin embargo, existen otros procesos sociales tales como la creación y el funcionamiento de redes sociales, las consecuencias de desigualdades en capital social, efectos acumulativos y no intencionales de intervenciones gubernamentales, agrupaciones de intermediarios y de los enclaves étnicos, etc., que también aparecen como importantes en la movilización de trabajadores en busca de nuevos horizontes en otras fronteras ${ }^{11}$.

Otro dato relevante en el análisis de los procesos migratorios, como agrega Castels, en el artículo anotado: "Por lo general, los movimientos migratorios surgen de la existencia entre los países de origen y los de acogida de unos lazos anteriores que pueden tener que ver con la colonización, la influencia política, el comercio la inversión o las relaciones culturales" $"$. Este antecedente, para el caso que nos interesa observar, es sin lugar a dudas muy importante, ya que la migración dentro del continente, tiene ciertamente una importante raíz en varios de estos factores, como ser: los procesos de independencia, los tratados de intercambio y la tradición cultural compartida. De todas formas, el flujo de población que se moviliza entre los distintos países del globo, 
que ha crecido de manera numerosa después de la década de los ochenta, no puede entenderse si no se integra al análisis la globalización, que como proceso, produce la eliminación de las fronteras económicas y la transnacionalización y aumento de las corrientes de comunicaciones, también implica, la universalización de algunos principios como el de los derechos humanos, todo ello afecta de manera muy importante la migración y por lo tanto requiere también de un análisis particular.

\section{LA GLOBALIZACIÓN Y SU RELACIÓN CON LAS MIGRACIONES}

El mundo actual se caracteriza por lo que se conoce como mundialización o globalización, es decir, la creciente gravitación de los procesos económicos, sociales y culturales de carácter mundial en los ámbitos nacional y regional. Aunque no se trata de un proceso nuevo, puesto que tiene raíces históricas bien profundas, los cambios en términos de espacio y de tiempo provocados por la revolución de las comunicaciones y la información le han dado nuevas dimensiones, que representan transformaciones cualitativas con respecto al pasado.

Como lo señala la Cepal: "En el último cuarto del siglo XX se consolidó una tercera fase de globalización, cuyos principales rasgos son la gradual generalización del libre comercio, la creciente presencia en el escenario mundial de empresas multinacionales que funcionan como sistemas de producción integrada,... y el acceso masivo de información y comunicaciones." 12

Los autores de la globalización la describen multidimensional y la reconocen como un proceso histórico, que comienza con la conquista de los océanos y que produce un fenómeno de extensión de fronteras, no solo geográficas, sino que cognitivas, comerciales, sociales, culturales, etc.

En el contexto descrito, la globalización es una modificación significativa de las formas de vida de los seres humanos, que contempla: la aceptación de un conjunto de reglas económicas para el mundo entero orientadas a maximizar los beneficios y la productividad por medio de la universalización de los mercados y la producción; innovaciones tecnológicas y cambios organizacionales centrados en la flexibilización y la adaptabilidad; reducción del estado de bienestar, privatización de los servicios sociales y la difusión de pautas culturales comunes, junto al desarrollo de nacionalismos, conflictos culturales y movimientos sociales ${ }^{11}$.

Las implicancias de la globalización en los movimientos migratorios son fáciles de suponer, especialmente en relación con las facilidades de comunicación y de transporte. Pero existen otros aspectos que es necesario considerar y que se relacionan también con la globalización. Se trata del impacto que ha provocado entre los ciudadanos la universalización de la cultura de la imagen y de la promoción del interés privado como forma de desarrollo. Estos dos aspectos, son trascendentales para entender la migración como un proceso destinado a la satisfacción de las necesidades de consumo, en una sociedad mundial altamente homogeneizada y que estimula el consumo individual, como un valor de integración a la gran comunidad internacional.

Por esta razón, la globalización de la cultura lleva a la exigencia del derecho al consumo por parte de las personas. El hombre de hoy es un cosmopolita que exige movilidad social o simulada. Esto quiere decir que si no tiene movilidad social real, puede sentirse bien accediendo a los lugares de consumo, como shopping, centers o supermercados, aunque sea sólo para compras pequeñas, o para pasear y consumir vidrieras. Por otra parte, la globalización de la economía hace que las personas se desplacen al "sabor de las oportunidades" ofrecidas por el capital internacional, y el avance tecnológico en el área de las comunicaciones hace posible desplazamientos a grandes distancias, manteniendo un contacto impensable hasta hace dos décadas ${ }^{13}$.

Cada uno de estos antecedentes para el análisis del caso de las migraciones dentro de nuestro continente, que tienen como país de destino a Chile, son capitales para poder comprender sus causas. Chile forma parte de este subcontinente y se encuentra atado a un pasado común, desde la independencia. 
Por ello, culturalmente el continente tiende a concebirse como una unidad, que facilita los flujos migratorios, pero, además, agrega un buen desempeño en su crecimiento económico y una imagen internacional positiva.

\section{CHILE EN EL CONCIERTO LATINOAMERICANO}

Chile como otros países del continente ha forjado su historia con la ayuda de la migración extranjera. Hasta finales del siglo pasado propició una migración selectiva para la ocupación y colonización de territorios. En la actualidad, es posible apreciar zonas con una marcada presencia de ciudadanos cuyas generaciones anteriores provinieron de otras latitudes, como es el caso de los alemanes en el sur continental, los yugoslavos en Punta Arenas y Antofagasta y los chinos en el norte del país.

La importancia de estas migraciones es muy grande, se aprecia en el valor asignado y traducido en iconos como las estatuas a los migrantes en distintos puntos de nuestro país, igualmente se puede palpar este valor, en la penetración que las culturas de estas otras tierras han producido entre nosotros y que se materializa en la asimilación de prácticas de trabajo, la dominación de ciertas empresas y el uso de parte de nuestros nacionales, de prácticas cotidianas de estos migrantes, como ser bailes y comidas.

Posteriormente, hasta la década de los noventa, sólo se observa un movimiento migratorio de los europeos después de la segunda guerra mundial.

En los noventa se produce un cambio, Chile acelera su inserción al mundo globalizado, con una mayor apertura de sus mercados y la aceptación de los modelos económicos internacionales en materia de aranceles, restricción del gasto público, fomento de las exportaciones y participación de acuerdos internacionales y subregionales de integración.

A su vez, con el inicio de la transición democrática, el país hace grandes esfuerzos por conectarse al mundo, del que a causa de la dictadura había sido aislado; asistiendo a citas internacionales, aceptando invitaciones a otras naciones y promoviendo una imagen de nación estable y exitosa.

Por otra parte, efectivamente exhibe indicadores reales de mejoras en su desarrollo. En primer lugar, se puede mostrar una transición democrática estable. La dictadura militar es derrotada en un proceso incruento y entrega el gobierno, aceptando su pérdida. El país es gobernado por una coalición de gran representación, que integra amplios sectores ligados al centro político y a la izquierda democrática. Los temas éticos sin ser resueltos, son abordados en el itinerario del nuevo gobierno y la nación retoma una senda que le dá legitimidad en el concierto latinoamericano y mundial.

En el terreno económico, de acuerdo a cifras de la Cepal, extraídas de su informe "Una década de luces y sombras" ${ }^{14}$, Chile destaca en algunos indicadores. Si se compara la tasa promedio anual del producto interno bruto total y por habitante de la década 1990-1999, muestra un promedio de PIB total de $6 \%$, mientras que América Latina y el Caribe exhiben una cifra de $3,2 \%$. Si se compara el producto interno bruto por habitante Chile muestra un crecimiento de $4,4 \%$ y el resto, tiene un crecimiento promedio de $1,4 \%$.

Cuando los números se refieren a los coeficientes de exportación, considerando los valores promedios con respecto al PIB en dólares del 1995, para el año 1999 Chile muestra 34,8 contra 19,8 que corresponde a la cifra de toda América Latina.

Por último, si se analiza los flujos de capital accionario en cifras netas hacia la región desde el extranjero, nuestro país figura dentro de los primeros lugares, compartiéndolos con Argentina y Brasil, países que históricamente fueron privilegiados. Cabe agregar, que también en términos de inversión intrarregional, Chile fue una importante figura, ya que ocupó importantes plazas en inversiones en energía y empresas de supermercados y multitiendas, entre otras. Todas estas cifras, no revelan los problemas que se perciben en el interior, en relación a los límites de la democracia, la exclusión social y la desigualdad, que están en paralelo con los resultados anotados por la Cepallit, y por ello, 
tampoco forman parte de la imagen en el exterior. Por el contrario, somos visualizados como una nación emprendedora, con múltiples oportunidades para el interés privado.

En este contexto, sumado a la igualdad del idioma y la tradición de una identidad continental, Chile se transformó en un país atractivo para los latinoamericanos, con una realidad distinta; afectados por la violencia, la inseguridad personal, las inestabilidades políticas y las situaciones de crisis económicas. Si a lo anterior, se agrega que existe un acuerdo internacional como el Convenio Andrés Bello, que facilita el desplazamiento de los profesionales, se puede entender la corriente migratoria de médicos.

Por ello, como señala Bazo: "Hacia mitad de la década, los migrantes comienzan a notarse en la escena del país, son tema de conversación $\mathrm{y}$ aparecen en los medios a partir de algunos hechos puntuales, la situación de los refugiados políticos, el conflicto suscitado por la incorporación de médicos cubanos, peruanos y ecuatorianos en la atención primaria de salud" ${ }^{15}$. De acuerdo con registros de la Corporación Ayun, que agrupa a los migrantes del continente, el país habría recibido durante la década de los noventa cerca de 200.000 migrantes latinoamericanos, habiendo llegado casi la mitad en los dos últimos años de la década del noventa.

En estas condiciones, Chile vive por primera vez en su historia, un fenómeno migratorio de estas proporciones, un volumen significativo no previsto ni promovido por el Estado y que no cuenta con un sistema legal que de cuenta de esta nueva realidad. El sistema legal de extranjería data de la década del cincuenta y es reelaborado durante el régimen militar, actualizándolo bajo los principios de la Doctrina de Seguridad Nacional, por lo tanto no adecuado a las necesidades actuales. Posteriormente se han hecho adecuaciones puntuales insuficientes. Para analizar en forma específica el tema de la migración de los profesionales médicos; es preciso agregar como antecedentes del panorama nacional dos hechos más, que se refieren al tema de la salud. El primero, dice relación con la fuerte presencia continental del país en el terreno de los servicios de atención; puesto que desde mediados del siglo pasado, producto del gran desarrollo de la salud pública tanto, en términos concretos en cobertura de servicios y organización, como en escuela de formación, atrajo la atención no solo de los países vecinos sino que de todo el mundo ${ }^{16}$. El segundo hecho, tiene que ver con lo que ocurre en la política de salud durante la década de los noventa. Al respecto, el primer gobierno de la concertación se plantea como objetivos primordiales en salud, el aumento de las inversiones en infraestructura y el fortalecimiento de la atención primaria. Estos dos objetivos produjeron una ampliación de las plazas de atención médica primaria en un $70 \%$ y a mediados de los 90 , estas plazas estaban desocupadas en un $40 \%{ }^{17}$, ya que no constituían ocupaciones atractivas para los médicos nacionales.

Por otra parte, a raíz de cambios en materias de salarios y otros en el mercado de la salud privada, también se generaron estímulos que potenciaron la migración de estos profesionales. Como señala el autor Francisco León en el trabajo citado: “. . . al contrario de lo que ocurría en materia cambiaria donde la moneda nacional continuaba valorizándose frente al dólar, las remuneraciones y costos del trabajo y de las atenciones de salud en Chile aumentaban con respecto a la de la mayoría de los países limítrofes. Esto genera la tendencia de hacer más atractivo a los agentes proveedores importar equipos, a los pacientes extranjeros atenderse en su país o en otros menos costosos, y a los profesionales del exterior venir a trabajar al país"2.

A lo anterior se agrega que la globalización, específicamente en relación con las políticas de salud, provoca también movimientos de los trabajadores de salud. A decir de la Organización Panamericana de la Salud: "La globalización aumenta la internacionalización de la producción y el consumo de bienes y servicios de salud, la inversión directa y extranjera en el sector de la salud y el movimiento de trabajadores de la salud entre los países". Y, en el mismo sentido, agrega a continuación: "Las reformas estructurales y sectoriales y la liberalización del comercio han conducido a un incremento de la comercialización de bienes y servicios de salud.... lo que plantea 
nuevos desafíos para la formulación de políticas de desarrollo del sector salud"18.

\section{CONCLUSIÓN}

En conclusión, se puede afirmar que la migración de médicos latinoamericanos a Chile en estas dos últimas décadas se produce con un convenio de los años sesenta, al servicio de la integración de nuestros países y la cooperación para el desarrollo conjunto, mientras que hoy este mismo acuerdo se usa en el marco de la globalización, donde destacan otros valores, como la competencia y la privatización de la vida cotidiana y de los servicios.

En efecto, como se ha señalado, el Convenio Andrés Bello se firma el año 1972, con un objetivo de integración política, que incorpora los procesos culturales de formación de profesionales y que no contempla una migración por motivos de mercado, ya que la atención de salud en los Estados nacionales en esos años, es en forma principal un derecho social. Por su parte, la globalización que favorece en general la migración por efectos directos sobre el transporte y las comunicaciones, suma impactos específicos en materia de la atención en salud, al estimular la creación de un mercado privado para esta atención y el desarrollo de un mercado internacional de servicios. Es decir, la globalización y los actuales modelos de apertura económica, donde predomina la integración de mercados, usan la herramienta de la integración política, que nuestros países firmaron en los sesenta, en función del valor cultural del intercambio de profesionales.

Los antecedentes presentados permiten dar fundamento efectivo a esta hipótesis. A partir de ésta, se puede señalar que el fenómeno comentado se da en el marco de un universo de globalización económica, cultural y política, como un proceso complejo, contradictorio e inevitable.

Complejo, por su carácter multidimensional, que como hemos visto depende de aspectos económicos, demográficos, culturales y sociales, entre otros.

Contradictorio, puesto que en el contexto de la globalización los movimientos de población migrando de un sitio a otro, reproducen otras contradicciones que este proceso de mundialización provoca, como la contradicción entre inclusión y exclusión, entre los roles del mercado y del Estado, entre las redes y el yo, lo global y lo local, etc. ${ }^{19}$.

Inevitable, porque la dirección de los cambios que experimenta la humanidad son a favor del mayor acercamiento entre las naciones e incluso, hacia la integración de éstas en nuevas agrupaciones de ellas. Igualmente, el avance en las tecnologías del transporte y de la información, hacen que trasladarse sea mucho más fácil y económico. Por último, el proceso de homogenización de la cultura, también concurre en la dirección de hacer más accesible la movilidad internacional.

\section{DISCUSIÓN}

Por todas estas razones, estamos frente a un fenómeno con el que las nuevas generaciones deberán contar como parte del paisaje futuro. Por lo mismo es indispensable mejorar las fórmulas que permitan la forma más útil para la mejor integración de estas poblaciones móviles a los países de destino, así como para que la alteración de los países de origen también sea la menor. Como dice Urzúa: "las migraciones internacionales son en nuestros días un componente inevitable de la agenda pública de muchos países, además del probable aumento que ellas han experimentado en las postrimerías del siglo que termina, los cambios en la dirección de las mismas y los problemas económicos, sociales y políticos que surgen en las diferencias étnicas, religiosas y culturales entre nativos y migrantes, han hecho que ellas no puedan estar ausentes de los debates políticos y que los países receptores ensayen distintas estrategias para revertir las tendencias o, al menos, disminuir los costos de un proceso que no es fácil cambiar en el corto o mediano plazo"11.

Así, la forma de asumir este fenómeno, que como se ha dicho es parte inevitable del paisaje, para que produzca los mejores resultados requiere, en primer lugar, aceptarlo y reconocerlo como positivo, sobre todo en nuestra situación, porque permite asegurar oferta de recursos profesionales, que en muchos casos no está 
disponible en el país. Sin embargo, también es indispensable establecer mecanismos que aseguren que su fin, cual es la atención médica de nuestra población, se realice de la mejor manera. En este sentido son útiles los mecanismos habituales de homologación y reconocimiento de estudios, vale decir, conocer los contenidos de las carreras de medicina de los países del continente y responder si tienen los requerimientos que se solicitan para el adecuado desempeño de estos profesionales.

Por otro lado, dado el valor que representan en aquellas especialidades deficitarias en nuestro país, es razonable que junto con estimular su venida, ésta se realice promoviendo la excelencia y la selección de especialistas deficitarios para ser distribuidos en todo el territorio de la nación.

\section{REFERENCIAS}

1.-Diversas publicaciones de prensa, especialmente del diario La Tercera, que se refieren al tema desde 1999 hasta la fecha.

2.-FRANCISCO LEÓN. Modernización y Comercio Exterior de los Servicios de Salud, Cepal, 2000.

3.-COLEGIO MÉDICO. 24 de agosto de 1999. (E1 Expreso, 10 de agosto de 2001).
4.- Información sobre el Convenio Andrés Bello en la página web homónima.

5.- PREBISCH R. Hacia una dinámica del desarrollo latinoamericano, FCE, México; 1971.

6.- ROSENTHAL G. "Treinta años de integración en América Latina: Un Examen Crítico" en Revista de Estudios Internacionales $\mathrm{N}^{\circ} 101$, Año XXVI, eneromarzo 1993.

7.- BOYE O. Trabas y Elementos Positivos para la Integración de América Latina, Informe Nro. 357. Asuntos Públicos, 5/12/2003.

8.- VANESA A. Integración Latinoamericana, Monografía, 2004.

9.- CASTELS B. Migración Internacional, 2000.

10.- URZÚA R. Migración Internacional, Ciencias Sociales y Políticas Públicas, CEPAL, 2000.

11.- CEPAL. La Migración Internacional y la Globalización, EN: Globalización y Desarrollo,

12.- CEPAL. 2002.

13.- BARRETO. 2002.

14.- CEPAL. Una década de luces y sombras, 2001.

15.- BAZO R. Migración en Chile, Le Monde Diploimatique: 2001.

16.- SIGERIST H. Historia y Sociología de la Medicina FCE 1973.

17.- MINISTERIO DE SALUD. 1996.

18.- OPS. Mercado de la Salud y Mercosur, 2003.

19.- CASTELS S. Migración internacional al comienzo del Siglo XXI, Boletín Cepal, 1997. 